\title{
Impact $\mathrm{H} \alpha$ line polarization and return current
}

\author{
M. Karlický ${ }^{1}$ and J. C. Hénoux ${ }^{2}$ \\ 1 Astronomical Institute, Academy of Sciences of the Czech Republic, 25165 Ondřejov, Czech Republic \\ 2 Observatoire de Paris, DASOP/UMR8645, place Jules Janssen, 92195 Meudon Principal Cedex, France \\ e-mail: henoux@mesiob.obspm.fr
}

Received 26 October 2001/ Accepted 7 December 2001

\begin{abstract}
Electron beams, bombarding the dense chromospheric layers during solar flares, carry electric currents which need to be neutralized by so-called return currents. Return currents are formed by background plasma electrons having an anisotropic velocity distribution. Thus they can generate impact $\mathrm{H} \alpha$ line polarization. First, a numerical method of computation of the impact $\mathrm{H} \alpha$ line polarization for an arbitrary electron distribution function is presented. Then the polarization due to return current electrons associated with beam electrons is computed. For low electron beam fluxes, the return current is low and the polarization is only due to the electron beam, i.e. it is perpendicular to the electron beam direction and it reaches $-8.0 \%$. Increasing the return current and the beam flux leads to a change of orientation of the polarization by $90^{\circ}$ and the polarization degree can even reach a maximum of $+22.4 \%$. But this change and the maximum of the polarization require very high electron beam fluxes of $1.79 \times 10^{12}$ and $4.8 \times 10^{12} \mathrm{ergs}^{-2} \mathrm{~s}^{-1}$, respectively. Therefore plasma processes, which can reduce the high-energy flux requirement for the polarization change observations, are briefly discussed.
\end{abstract}

Key words. Sun: flare - plasmas - polarization

\section{Introduction}

The chromospheric hydrogen $\mathrm{H} \alpha$ and $\mathrm{H} \beta$ Balmer lines have been found to be linearly polarized in solar flares. $\mathrm{H} \alpha$ polarization was observed in $2 \mathrm{D}$ filtergrams obtained with a monochromatique Lyot filter centered on the $\mathrm{H} \alpha$ line center with a 0.75 bandpass (Vogt \& Hénoux 1996). Independently, the wavelength dependence of the linear polarization along $\mathrm{H} \alpha$ and $\mathrm{H} \beta$ line profiles was derived from spectroscopic observations, taken at various positions in a few solar flares (Firstova \& Boulatov 1996; Hénoux et al. 2001). Recently, an unusual behavior of the Stokes parameter profiles along the dispersion observed during a flare with a powerful surge was reported by Firstova \& Kashapova (2001).

In solar flare $\mathrm{H} \alpha$ filtergrams, the highest number of pixels emitting polarized $\mathrm{H} \alpha$ radiation is observed near the time of the maximum of soft X-ray emission. The degree of linear polarization reaches 5 to $10 \%$ and the direction of polarization in most of the flaring region is the flare to disk center direction (Vogt \& Hénoux 1999). In $\mathrm{H} \alpha$ and $\mathrm{H} \beta$ flare spectra, obtained during the flare progressive phase (Hénoux et al. 2001), the degree of polarization can be as high as $15 \%$ in the $\mathrm{H} \beta$ line center and does not exceed $5 \%$

Send offprint requests to: M. Karlický, e-mail: karlicky@asu.cas.cz in the $\mathrm{H} \alpha$ line center. Both radial and tangential directions of polarization are present at different locations.

The observed polarization indicates that during solar flares energetic particles, electrons, protons or ions, with an energy high enough to collisionally excite the hydrogen $\mathrm{H} \alpha$ line, are present in the solar chromosphere with an anisotropic velocity distribution function.

In the absence of hard X-ray emission, hecta keV protons have been suggested as the origin of the observed impact polarization. When depolarizing processes are taken into account, a polarization degree of 4 to $5 \%$ in $\mathrm{H} \alpha$ can be expected from proton excitation assuming that the atmosphere can be represented by the VAL F model (Vogt et al. 1997, 2001). However, for a denser and hotter chromosphere like the one described by the MAVN model F1, the resulting polarization drops to values close to $1 \%$.

For identical velocity distribution anisotropies, electrons with energies in the range $12-50 \mathrm{eV}$ are expected to lead to a higher polarization degree than $200 \mathrm{keV}$ protons (Werner \& Schartner 1996). Usually, such low energy electrons are expected to have isotropic velocity distribution functions and therefore not to produce significant polarization. However, in cases where electrons with anisotropic distribution functions are present at chromospheric levels, as in the case of the existence of a return current, a higher polarization degree may be generated. This case is investigated in this paper. 


\section{Impact $\mathrm{H} \alpha$ line polarization due to a cloud of superthermal electrons}

For purposes of the following numerical computations we present analytical formulas for an individual superthermal electron as well as for a cloud of these electrons.

\subsection{The impact $\mathrm{H} \alpha$ line polarization due to an individual superthermal electron}

The monochromatic radiation emitted by an atom collisionally excited by a particle of energy $E$ may be linearly polarized (Percival \& Seaton 1959). The degree of linear polarization $P(\beta, E)$ is usually defined as

$P(\beta, E)=\left(I_{\|}-I_{\perp}\right) /\left(I_{\|}+I_{\perp}\right)$,

where $I_{\|}$and $I_{\perp}$ are respectively the intensities of the vibrations parallel and perpendicular to the plane defined by the particle trajectory and the line of sight and $\beta$ is the angle between these two directions.

$P(\beta, E)$ is related to the maximum of polarization observable at $90^{\circ}$ from the particle propagation direction, $P\left(90^{\circ}, E\right)$, by

$P(\beta, E)=P\left(90^{\circ}, E\right) \frac{\sin ^{2} \beta}{1-P\left(90^{\circ}, E\right) \cos ^{2} \beta}$.

$P\left(90^{\circ}, E\right)$ is a function of the nature of the particle, electron, proton or ion and of the particle energy. $P\left(90^{\circ}, E\right)$ changes of sign at a turnover energy $E_{0}$. For the $\mathrm{H} \alpha$ line, $E_{0}$ is about $200 \mathrm{eV}$ for electrons and $200 \mathrm{keV}$ for protons. For electrons the energy dependence of $P\left(90^{\circ}, E\right)$ used for numerical computation has been approximated by the curve given in Fig. 1. In order to generate impact $\mathrm{H} \alpha$ polarization, an electron needs to have an energy above $12.1 \mathrm{eV}$, which is the excitation potential between hydrogen atom 1 and 3 levels.

\subsection{Impact $\mathrm{H} \alpha$ line polarization due to a cloud of electrons}

In order to derive the orientation of the linear polarization and the polarization degree for any electron velocity distribution function, a numerical code has been built where this distribution function has been represented as a sum of numerical electrons. A coordinate system $X Y Z$ was selected as the system of reference for an observer collecting photons propagating along the $Z$ direction, as shown in Fig. 2. The $\mathrm{H} \alpha$ line linear polarization resulting from impact excitation, at location $\mathrm{O}$, of neutral hydrogen atoms by a moving electrons is defined by the Stokes parameters $Q_{X Y}$ and $U_{X Y}$ defined as

$$
\begin{aligned}
Q_{X Y} & =\frac{I_{X}-I_{Y}}{I_{X}+I_{Y}} \\
U_{X Y} & =\frac{I_{X_{90}}-I_{Y_{90}}}{I_{X_{90}}+I_{Y_{90}}},
\end{aligned}
$$

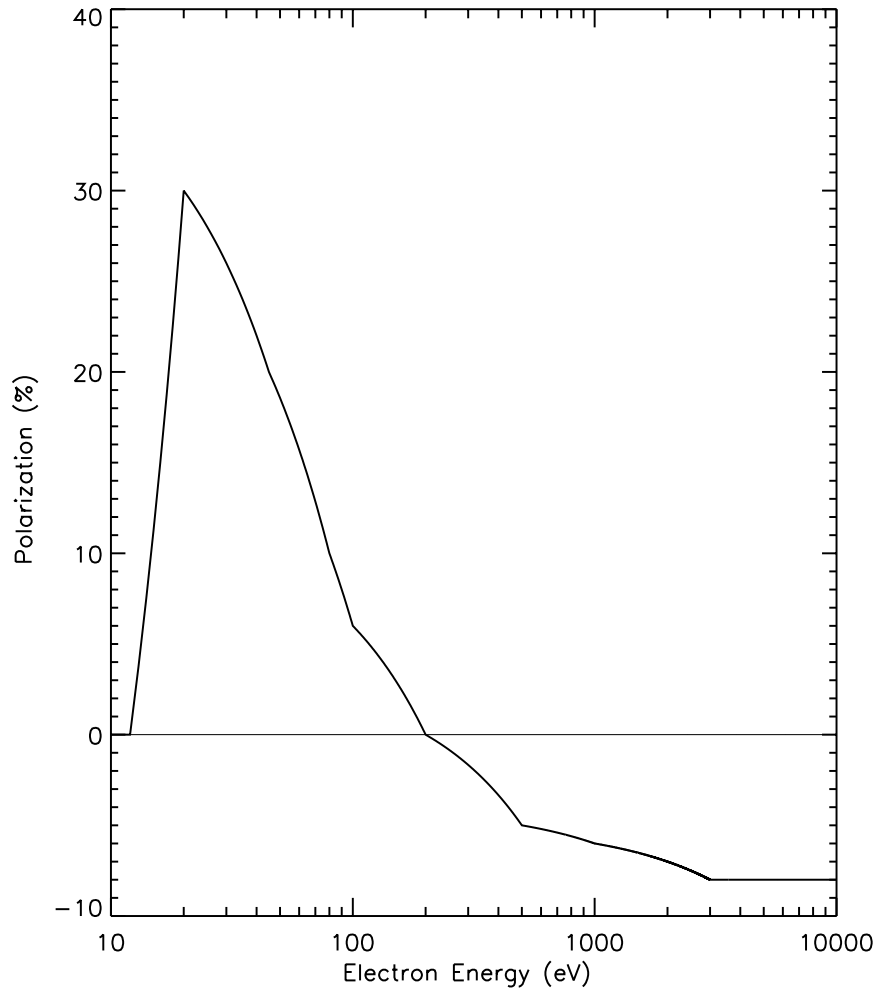

Fig. 1. $P\left(90^{\circ}, E\right)$ for the $\mathrm{H}_{\alpha}$ line for electron impact excitation as function of the electron energy used in the numerical computations. The polarization is linearly interpolated in energy intervals between specific values taken from Hénoux \& Vogt (1998).

where the $X_{90} Y_{90} Z$ coordinate system has been derived from the $X Y Z$ coordinate system by a $90^{\circ}$ rotation around the $Z$ axis.

In the present case $Q_{X Y}$ and $U_{X Y}$ can be obtained by adding the Stokes parameters $Q_{X Y}^{i}$ and $U_{X Y}^{i}$ of the radiation generated by every individual electron $i$ forming any non-thermal electron velocity distribution function, i.e.

$Q_{X Y}=\sum_{i=1}^{i=n} Q_{X Y}^{i}$
$U_{X Y}=\sum_{i=1}^{i=n} U_{X Y}^{i}$,

where summation is made over all electrons $i$.

The electron direction of propagation and the line of sight define a plane. This plane naturally leads to take for the $x$ and $y$ directions the directions parallel and perpendicular to this plane.

Calling $\alpha$ the angle between the $O x(O y)$ and $O X$ $(O Y)$ axes, the Stokes parameters, in the two $x y Z$ and $X Y Z$ systems of reference, of the radiation generated by an individual electron are related by the usual law for rotation of axis:

$Q_{X Y}^{i}=Q_{x y}^{i} \cos 2 \alpha+U_{x y}^{i} \sin 2 \alpha$

$U_{X Y}^{i}=-Q_{x y}^{i} \sin 2 \alpha+U_{x y}^{i} \cos 2 \alpha$. 


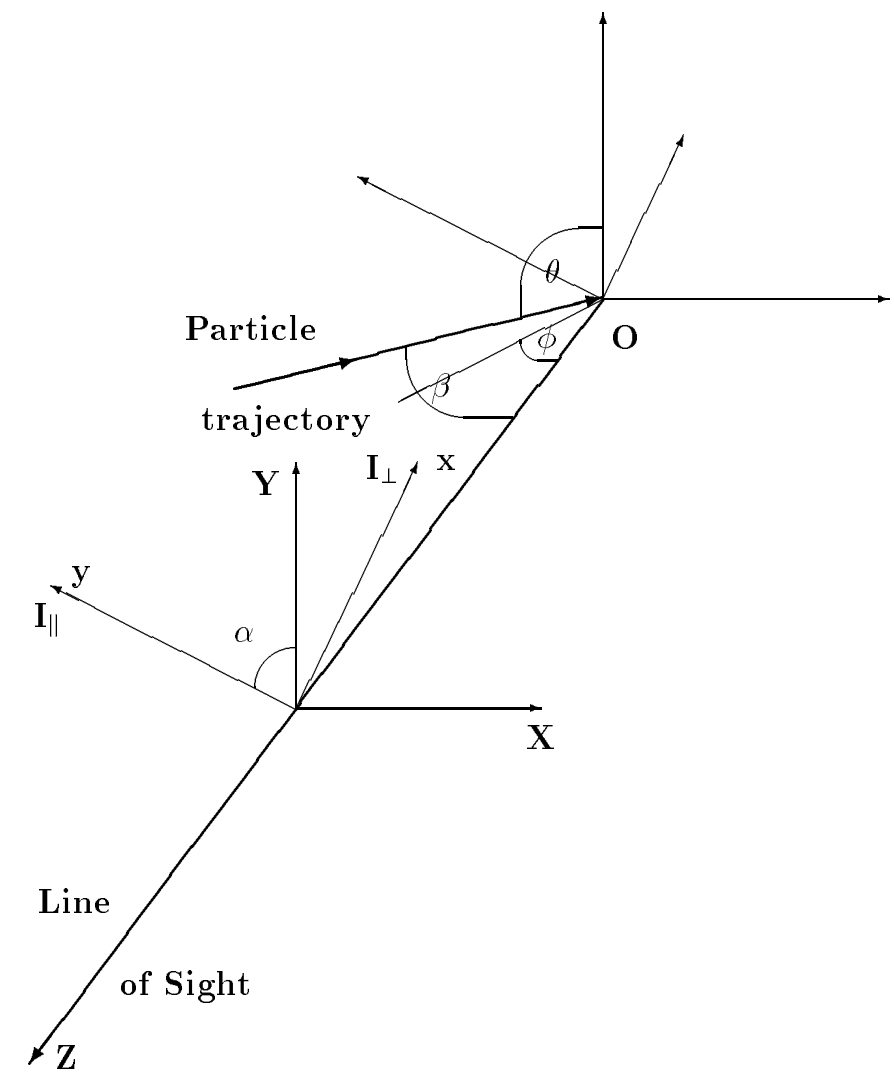

Fig. 2. Coordinate systems defining the Stokes parameters of impact radiation generated by an ensemble of electrons moving in any direction.

In the $x y Z$ system of reference:

$Q_{x y}^{i}=P(\beta, E)^{i} I^{i}$,

where $I^{i}=\left(I_{x}^{i}+I_{y}^{i}\right)$ and $U_{x y}^{i}=0$. Consequently

$Q_{X Y}^{i}=P(\beta, E)^{i} I^{i} \cos 2 \alpha^{i}$

$U_{X Y}^{i}=-P(\beta, E)^{i} I^{i} \sin 2 \alpha^{i}$

and

$Q_{X Y}=\sum_{i=1}^{i=n} P(\beta, E)^{i} I^{i} \cos 2 \alpha^{i}$

$U_{X Y}=\sum_{i=1}^{i=n}-P(\beta, E)^{i} I^{i} \sin 2 \alpha^{i}$.

The net degree of polarization $\mathcal{P}$ and the angle $\Psi$ between the main direction of polarization and the $O X$ axis are given respectively by

$\mathcal{P}=\sqrt{Q_{X Y}^{2}+U_{X Y}^{2}} / \sum_{i=1}^{i=n} I^{i}$

and

$$
\begin{aligned}
& \sin 2 \Psi=U_{X Y} / \sqrt{Q_{X Y}^{2}+U_{X Y}^{2}} \\
& \cos 2 \Psi=Q_{X Y} / \sqrt{Q_{X Y}^{2}+U_{X Y}^{2}}
\end{aligned}
$$

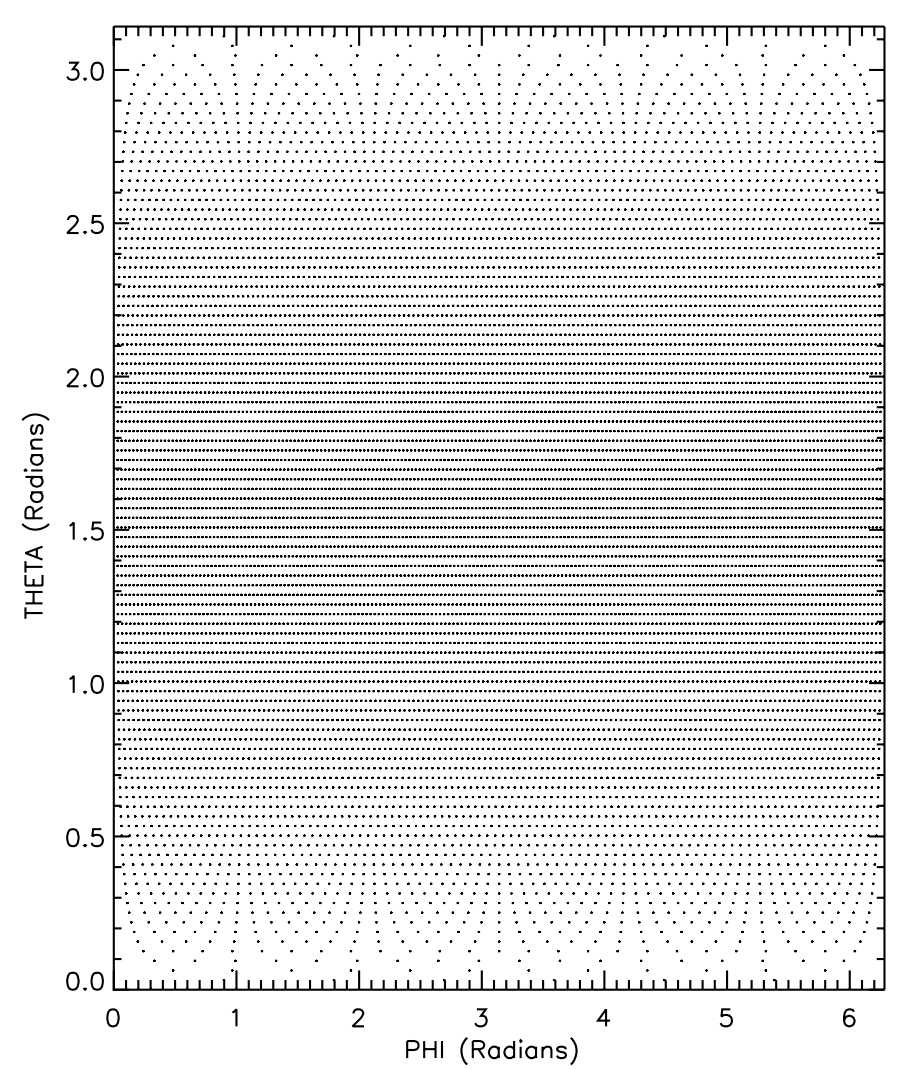

Fig. 3. Initial spherically symmetrical distribution of numerical electrons in the $\Theta-\Phi$ space.

\section{Return current and impact linear $\mathrm{H} \alpha$ line polarization}

It is known that electrons accelerated and heated during the impulsive phase and also during the post-flare phase bombard the dense chromospheric layers of the flare atmosphere. This flux of electrons may carry a huge electric current. So, in response, the background plasma electrons neutralize this current by generating a return current (Hoyng et al. 1976; van den Oord 1990). A simple equation for the return current $j_{\mathrm{R}}$ relate the electron velocities in the beam and in the return current:

$j_{\mathrm{R}}=e n_{\mathrm{e}} v_{\mathrm{R}}=-e n_{\mathrm{b}} v_{\mathrm{b}}=j_{\mathrm{b}}$,

where $e$ is the electron charge, $n_{\mathrm{e}}, n_{\mathrm{b}}$ are the electron densities of the background plasma and electron beam, $v_{\mathrm{R}}$, $v_{\mathrm{b}}$ are the electron velocities of the return and bombarding $\left(j_{\mathrm{b}}\right)$ currents.

The return current is formed by all background plasma electrons having both the thermal velocity plus a specific return current velocity. For numerical computations, let us simulate the distribution function of the return current electrons by a cloud of numerical electrons. First, for a constant velocity (corresponding to $20 \mathrm{eV}$ electrons), a spherically symmetrical distribution was generated for the direction angles $\Theta$ and $\Phi$ (Fig. 3). The $4 \pi$ solid angle space was divided into a given number of elementary elements carrying an equal number of numerical electrons. Then it was assumed that this sphere in velocity space moves 


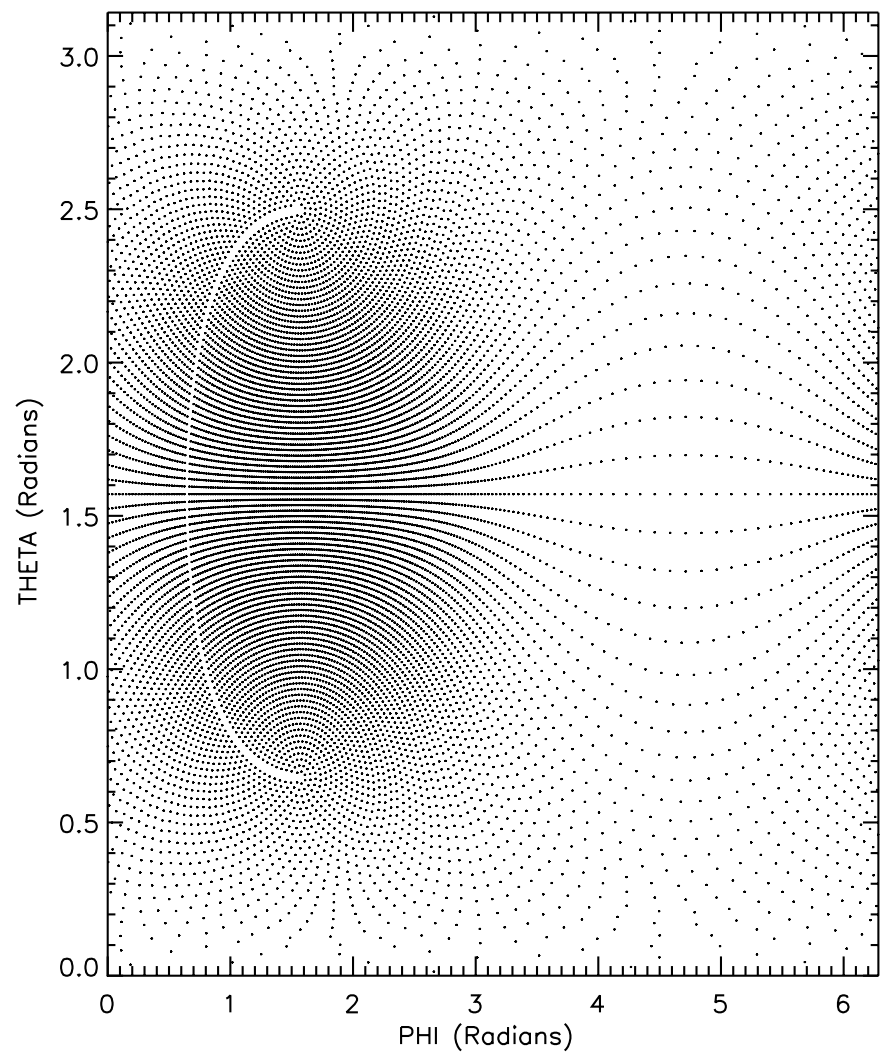

Fig. 4. Distribution of numerical electrons in the $\Theta-\Phi$ space when a return current with speed $v_{\mathrm{R}}=2 \times 10^{6} \mathrm{~m} \mathrm{~s}^{-1}$ is added to the spherically symmetrical velocity distribution.

with a given specific velocity corresponding to the return current electron speed. This causes the direction of the return current electrons to change, as seen in Figs. 4 and 5. In these figures the distribution of electron directions in the $\Theta-\Phi$ space for a return current electron speed $v_{\mathrm{R}}$ equal to $2 \times 10^{6}$ and $2 \times 10^{7} \mathrm{~m} \mathrm{~s}^{-1}$ (the gaps in Fig. 4 and in Fig. 6 are due to the fact that the $4 \pi$ sphere is not fully covered by all elementary solid angle elements. Such small gaps have no significant influence on the results). The speed of the return current electrons is directed along the $O X$ axis. By adding the return current speed to the thermal speed, the energies of the electrons also change, as seen in Fig. 6, where the distribution of energy versus $\Theta$ angle is depicted.

A full description of the return current electrons is made by using 41 spheres in velocity space (in the energy interval 0-25 eV) of radius the absolute electron velocity. All spheres have different numerical weights, since they correspond to a different number of real electrons. These weights are selected in agreement with a thermal (Maxwellian) distribution of a thermal energy of $1 \mathrm{eV}$.

By simulating the velocity distribution of the return current electrons, the polarization of the $\mathrm{H} \alpha$ line can be computed. For a 90 degree view angle, due to the relation between the return current velocity and the beam electrons speed (see Eq. (15)), the polarization degree generated by an electron beam plus its associated return current depends only on the return current velocity.

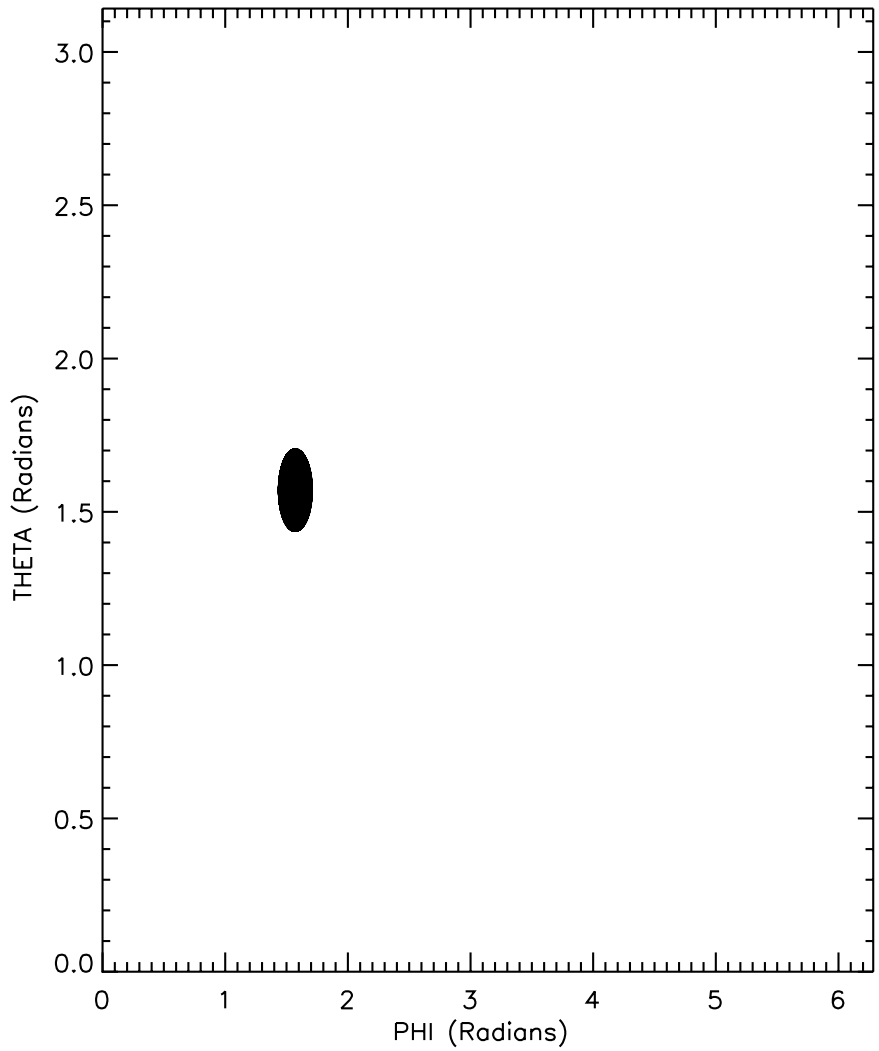

Fig. 5. Distribution of numerical electrons in the $\Theta-\Phi$ space when a return current with speed $v_{\mathrm{R}}=2 \times 10^{7} \mathrm{~m} \mathrm{~s}^{-1}$ is added to the spherically symmetric velocity distribution.

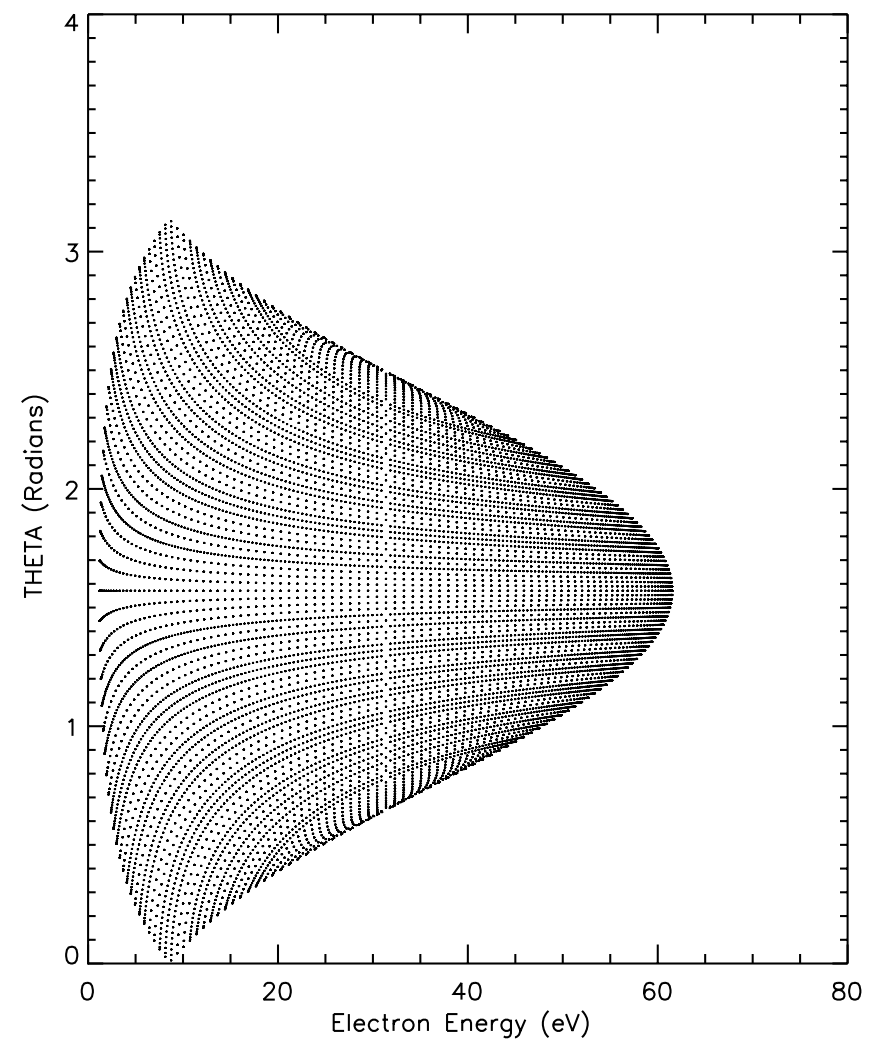

Fig. 6. Energy distribution of electrons as a function of the $\Theta$ angle for the case presented in Fig. 4. The initial energy in the spherically symmetric case was $20 \mathrm{eV}$. 


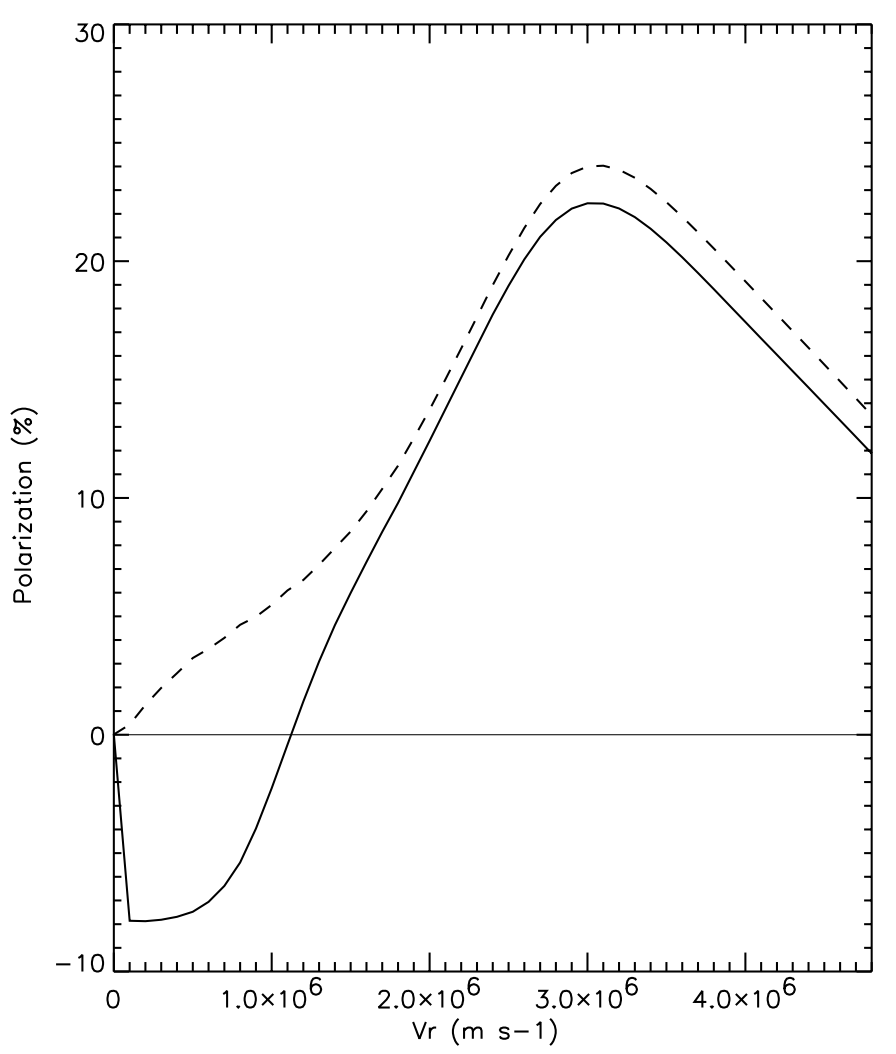

Fig. 7. Polarization degree as a function of the return current electrons speed. The dashed line corresponds to the case where only the return current electrons are considered. The solid line expresses polarization when the effect of bombarding electrons is also included.

Ignoring the polarization generated by the electron beam at the origin of the return current, such a dependence is represented by a dashed line in Fig. 7. This polarization is positive (a positive polarization degree corresponds to a polarization vector directed along the beam and return current propagation direction and negative values correspond to a direction perpendicular to this direction). By increasing the return current velocity, the polarization degree is found to increase monotonically from zero, for a null return current speed, up to a maximum of $24 \%$ for $v_{\mathrm{R}}=3 \times 10^{6} \mathrm{~m} \mathrm{~s}^{-1}$ and then to decrease. Beam electrons on the other hand generate negative polarization. In order to evaluate the net polarization signal (solid line in Fig. 7) that results from the sum of the return current and beam contributions, a beam of $10 \mathrm{keV}$ monoenergetic electrons was assumed. The relative density of the beam $\left(n_{\mathrm{b}} / n_{\mathrm{e}}\right)$ was derived by using Eq. (15). The polarization, null for zero return current, becomes negative and drops down to $-8 \%$ as soon as the return current electron speed rises. The polarization changes sign at about $v_{\mathrm{R}}=1.12 \times 10^{6} \mathrm{~m} \mathrm{~s}^{-1}$, increases to a maximum of $22.4 \%$ at about $v_{\mathrm{R}}=3 \times 10^{6} \mathrm{~m} \mathrm{~s}^{-1}$, the velocity at which the bulk of return current electrons reaches energies of $20 \mathrm{eV}$, and decreases thereafter.

For comparison, the polarization was also computed for a simple model where the thermal velocities were

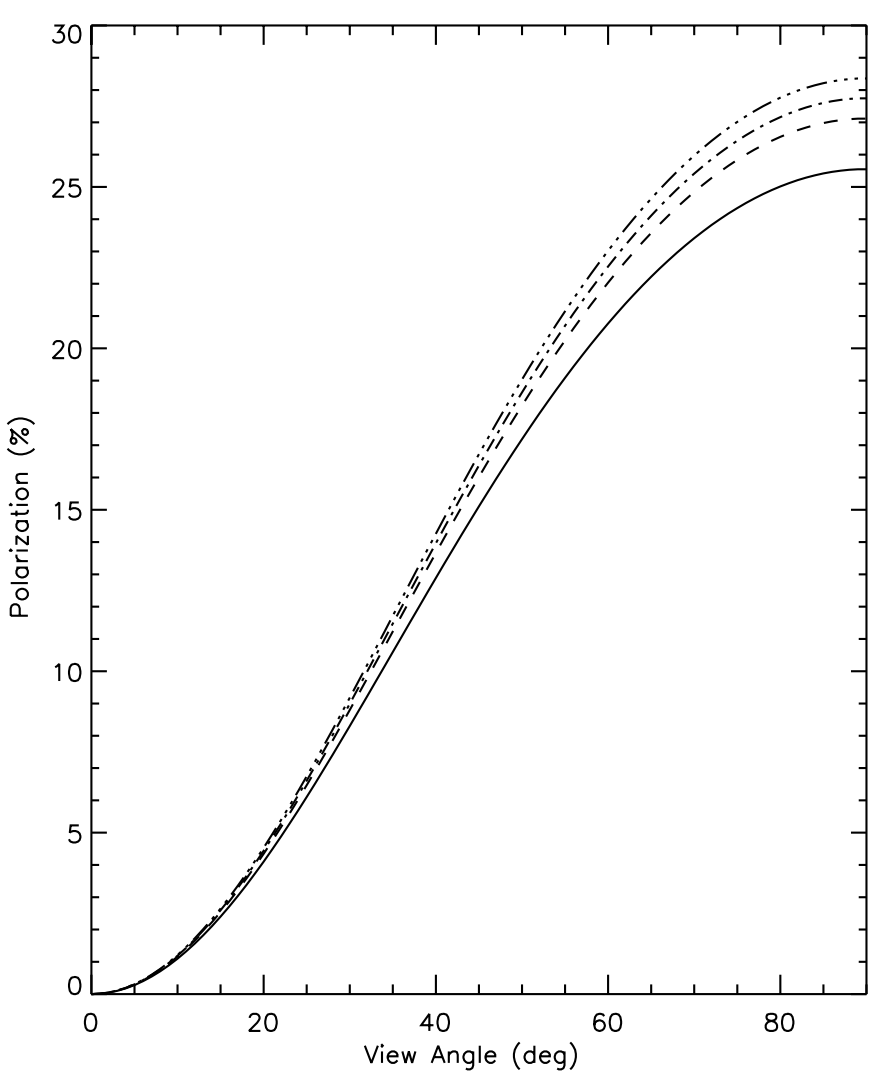

Fig. 8. Impact linear $\mathrm{H} \alpha$ line polarization degree as a function of the observing view angle for bombarding electrons with energies: $1 \mathrm{keV}$ (full line), $3 \mathrm{keV}$ (dashed line), $5 \mathrm{keV}$ (dash-dot line), and $10 \mathrm{keV}$ (dash-dot-dot-dot line).

Table 1. Selected energies of bombarding electrons and derived parameters of return and bombarding currents in simple return current model. The energy of return current electrons is chosen as $20 \mathrm{eV}$.

\begin{tabular}{|l|l|l|}
\hline$E(\mathrm{keV})$ & $v_{\mathrm{b}} / v_{\mathrm{R}}$ & $n_{\mathrm{R}}: n_{\mathrm{b}}$ \\
\hline 1 & 7.07 & $1000: 141$ \\
\hline 3 & 12.25 & $1000: 82$ \\
\hline 5 & 15.81 & $1000: 63$ \\
\hline 10 & 22.36 & $1000: 45$ \\
\hline
\end{tabular}

neglected in the return current distribution. In this model, the polarization was computed with two counterstreaming beams (the return current and bombarding beams). The energy of return current electrons was considered to be $20 \mathrm{eV}$ (energy at which the polarization degree has a maximum - see Fig. 1) and the polarization for 4 selected energies of the bombarding electrons (Table 1) was computed as a function of the view angle (Fig. 8).

As expected, the polarization has a maximum at $90^{\circ}$ view angle. The polarization is only weakly dependent on the energies of the bombarding electrons, but decreases strongly towards the disk center (zero view angle). Comparing the polarization degrees in Fig. 7 and in Fig. 8, we see that the maximum polarization is smaller in Fig. 7 than in Fig. 8. This is due to the fact that in this case the thermal velocity dispersion was taken into account. 


\section{Discussion and conclusions}

The $\mathrm{H} \alpha$ polarization caused by an electron beam plus its associated return current was computed in this paper. Ignoring the depolarization process, discussed below, we showed that the polarization results from the opposing effects of the electron beam and of the return current. At a density $n_{\mathrm{e}}=10^{12} \mathrm{~cm}^{-3}$, the beam electron number fluxes for which the polarization changes sign and the one for which it reaches its maximum positive value $(22.4 \%)$ are respectively $1.12 \times 10^{20}$ and $3 \times$ $10^{20}$ electrons $\mathrm{cm}^{-2} \mathrm{~s}^{-1}$. The associated energy fluxes depend on the energies of the non-thermal electrons. A beam of monoenergetic electrons of energy $10 \mathrm{keV}$ will carry an energy flux as high as $4.8 \times 10^{12} \mathrm{ergs}^{-2} \mathrm{~s}^{-1}$ at the maximum of positive polarization. The change of polarization sign takes place for a still relatively high energy flux, $1.79 \times 10^{12} \mathrm{ergs} \mathrm{cm}^{-2} \mathrm{~s}^{-1}$. Below $1.79 \times 10^{12} \mathrm{ergs} \mathrm{cm}^{-2} \mathrm{~s}^{-1}$ the polarization is negative, being generated mainly or only by the beam electrons.

These estimations refer to local flux values at the level of $\mathrm{H} \alpha$ formation. Collisions and return current decelerating effects from the acceleration site to the $\mathrm{H} \alpha$ formation layer increase the energy flux requirements by at least one order of magnitude. Therefore, for return currents to explain the observed polarization, the energy requirement must be lowered. This can be done either by assuming lower electron densities in the $\mathrm{H} \alpha$ forming layer or by decreasing the individual energy of bombarding electrons. But here, there are limits.

Plasma effects can lower the number of background electrons participating in the return current formation, allowing us to obtain the return current electron velocity $v_{\mathrm{R}}=1.12 \times 10^{6} \mathrm{~m} \mathrm{~s}^{-1}$, where the polarization sign changes, for lower energy flux values. Namely, these electron velocities are much higher than the thermal speed of the chromospheric plasma $(\sim 1 \mathrm{eV})$. Then the associated currents are unstable for the Buneman instability; plasma waves are generated and the return current electrons interact with these waves; anomalous resistivity is generated (Heyvaerts 1981). This resistivity strongly depends on the distribution of plasma waves in the phase velocity space and the current electrons are trapped in these waves. Above some velocity threshold, electrons can have a higher mobility. In such a case, only this group of electrons can be involved in the return current. Then the number density of the electrons forming the return current can be lower than the total local electron number density $n_{\mathrm{e}}$. An important consequence is the associated reduction of the number and of the energy flux of bombarding electrons. However, these plasma processes are not still well understood; details of plasma microphysics need to be included.

Depolarization effects reduce the net polarization. This depolarization result from transfer of populations between the Zeeman excited states by the local protons with isotropic velocity distributions and by the radiation field. For low-energy protons the theoretical maximum of the polarization is only $10 \%$ (Hénoux \& Vogt 1998). When depolarizing effects are taken into account the expected polarization reaches 4 to $5 \%$ (Vogt et al. 2001). Such a value is not too different from the observed $\mathrm{H} \alpha$ polarization degree $(\sim 5 \%)$. The return current electrons have the advantage of generating, when ignoring depolarization effects, a higher polarization signal (up to $30 \%$ compared to $10 \%$ for protons). However, in the absence of a clear understanding of plasma effects, they seem to require too much energy. Models, e.g. with fragmented evaporative upflows (Fletcher \& Brown 1998), can also be considered.

Polarization observations made with an $\mathrm{H} \alpha$ filter have shown, for the only flare where both impulsive and progressive phases were observed, a change of the polarization orientation by $90^{\circ}$. The polarization was tangential during the impulsive phase, where high energy X-rays were observed, and became radial during the progressive phase (Vogt \& Hénoux 1999). Proton bombardment following high energy electron bombardment was proposed to explain such an observation. For this event the return current model would require the high energy electron flux to increase with time. That is not the case. More observations are needed. Polarization observations made with THEMIS (Hénoux et al. 2001) confirm the presence of linear polarization in two solar observed flares. The polarization degree reaches about $5 \%$ in $\mathrm{H} \alpha$ and more in $\mathrm{H} \beta$. At a given time both radial and tangential directions of polarization could be present in different places. Investigation of this phenomenan is underway.

Acknowledgements. M.K. acknowledges the support of Grant 205/00/1726 of GA ČR and Grants A3003202, A3003003 and S1003006 of GA AV ČR under the key project K2043105. Both authors thank the French National Center for Scientific Research (CNRS) and the Academy of Sciences of the Czech Republic. both authors thank the staff of the Solar Departments at Paris and Ondřejov Observatories for their support and kind hospitality.

\section{References}

Firstova, N. M., \& Boulatov, A. V. 1996, Sol. Phys., 164, 301 Firstova, N. M., \& Kashapova, L. K. 2001, A\&A, accepted

Fletcher, L., \& Brown, J. C. 1998, A\&A, 338, 737

Hénoux, J. C., \& Vogt, E. 1998, Plasma Scrip., 78, 60

Hénoux, J. C., Vogt, E., Chambe, G., \& Briand, C. 2001, Il Nuovo Cimento, in press

Heyvaerts, J. 1981, in Solar Flare Magnetohydrodynamics, ed. E. R. Priest (Gordon and Breach Science Publishers), 429

Hoyng, P., Brown, J. C., \& Van Beek, H. F. 1976, Sol. Phys., 48, 197

Percival, I. C., \& Seaton, M. J. 1959, Phil. Trans. R. S. London, A251, 113

van den Oord, G. H. 1990, A\&A, 234, 496

Vogt, E., \& Hénoux, J. C. 1996, Sol. Phys., 164, 345

Vogt, E., \& Hénoux, J. C. 1999, A\&A, 349, 283

Vogt, E., Sahal-Bréchot, S., \& Hénoux, J. C. 1997, A\&A, 324, 1211

Vogt, E., Sahal-Bréchot, S., \& Bommier, V. 2001, A\&A, 374, 1127

Werner, A., \& Schartner, K. H. 1996, J. Phys. B: At. Mol. Opt. Phys., 29, 125 